\title{
Aerobic glycolysis fuels platelet activation: small-molecule modulators of platelet metabolism as anti-thrombotic agents
}

\author{
Paresh P. Kulkarni, ${ }^{1 \dagger}$ Arundhati Tiwari, ${ }^{1 \dagger}$ Nitesh Singh, ${ }^{1}$ Deepa Gautam, ${ }^{1}$ Vijay \\ K. Sonkar, ${ }^{1}$ Vikas Agarwal ${ }^{2}$ and Debabrata Dash ${ }^{1}$ \\ ${ }^{1}$ Department of Biochemistry, Institute of Medical Sciences and ${ }^{2}$ Department of \\ Cardiology, Institute of Medical Sciences, Banaras Hindu University, Varanasi, Uttar \\ Pradesh, India \\ ${ }^{t} P P K$ and $A T$ contributed equally to this work.
}

Haematologica 2019

Volume 104(4):806-818

\section{ABSTRACT}

$\mathrm{P}$ latelets are critical to arterial thrombosis, which underlies myocardial infarction and stroke. Activated platelets, regardless of the nature of their stimulus, initiate energy-intensive processes that sustain thrombus, while adapting to potential adversities of hypoxia and nutrient deprivation within the densely packed thrombotic milieu. We report here that stimulated platelets switch their energy metabolism to aerobic glycolysis by modulating enzymes at key checkpoints in glucose metabolism. We found that aerobic glycolysis, in turn, accelerates flux through the pentose phosphate pathway and supports platelet activation. Hence, reversing metabolic adaptations of platelets could be an effective alternative to conventional anti-platelet approaches, which are crippled by remarkable redundancy in platelet agonists and ensuing signaling pathways. In support of this hypothesis, small-molecule modulators of pyruvate dehydrogenase, pyruvate kinase M2 and glucose-6-phosphate dehydrogenase, all of which impede aerobic glycolysis and/or the pentose phosphate pathway, restrained the agonist-induced platelet responses ex vivo. These drugs, which include the anti-neoplastic candidate, dichloroacetate, and the Food and Drug Administration-approved dehydroepiandrosterone, profoundly impaired thrombosis in mice, thereby exhibiting potential as anti-thrombotic agents.

Received: September 3, 2018.

Accepted: October 30, 2018.

Pre-published: October 31, 2018.

doi:10.3324/haematol.2018.205724

Check the online version for the most updated information on this article, online supplements, and information on authorship \& disclosures: www.haematologica.org/content/104/4/806

(C)2019 Ferrata Storti Foundation

Material published in Haematologica is covered by copyright. All rights are reserved to the Ferrata Storti Foundation. Use of published material is allowed under the following terms and conditions:

https://creativecommons.org/licenses/by-nc/4.0/legalcode. Copies of published material are allowed for personal or internal use. Sharing published material for non-commercial purposes is subject to the following conditions:

https://creativecommons.org/licenses/by-nc/4.0/legalcode, sect. 3. Reproducing and sharing published material for commercial purposes is not allowed without permission in writing from the publisher.

\section{Introduction}

Platelets play a prominent role in the pathophysiology of acute myocardial infarction and ischemic stroke, which are the major causes of mortality worldwide. ${ }^{1}$ Anti-platelet drugs remain the mainstay for the prevention of these catastrophic events. Nevertheless, the anti-platelet agents currently in vogue have limited efficacy in preventing thrombotic events without significantly raising bleeding risk. ${ }^{2}$ There is remarkable redundancy in potential agonists inducing platelet activation, as well as plasticity in the signaling paths downstream of these agonists. Patients on anti-platelet drugs that inhibit platelet responses to specific agonists continue to experience adverse thrombotic episodes, since other potential triggers and parallel signaling cascades can still activate platelets. Hence, it is vital to discover novel anti-platelet strategies to address these limitations.

In an intact vasculature, platelets are relatively inactive and are, therefore, described as 'resting'. However, they are extremely responsive to any breach in the vessel wall and are highly efficient in sealing the defect. Following a break in endothelium, platelets interact with, and adhere to, the now exposed subendothelial matrix proteins, such as collagen, through their cell surface receptors. These receptor-ligand interactions initiate signaling cascades that lead to: (i) a change in the shape of platelets from discoid to 'spiny-spheres', (ii) platelet-platelet aggregation through fibrinogen bridges connecting high-affinity integrins, and (iii) degranulation of platelet storage vesicles, the contents of which serve to amplify responses to vessel wall injury. A chain reaction of platelet activation and aggregate formation, as well as conversion of fibrinogen to insoluble polymers of fibrin, culminates 
in plugging of the defect in vasculature. This response is termed 'hemostasis'. A similar but more exaggerated response from platelets upon rupture of an atherosclerotic plaque leads to arterial thrombosis with potentially fatal consequences, such as myocardial infarction and ischemic stroke. ${ }^{3}$

Platelet responses to agonist stimulation, including adhesion, shape change, integrin activation, aggregation, exocytosis of granule contents and clot retraction are all energy intensive processes. ${ }^{4-7}$ Nevertheless, platelets accomplish these responses while trapped within the relatively impervious boundaries of a thrombus, with restricted access to nutrients and oxygen. We hypothesized that it is imperative for activated platelets within a thrombotic milieu to adapt their energy metabolism to these challenges in order to sustain and hold the thrombus together. We employed a high-resolution bioenergetics screen to establish aerobic glycolysis and the consequent flux through the pentose phosphate pathway (PPP) as the metabolic signature of agonist-stimulated platelets. Using small-molecule modulators - dichloroacetate (DCA), diarylsulfonamide (DASA-58) (DASA), and dehydroepiandrosterone sulphate (DHEA) - which target these pathways, we demonstrate that rewiring of metabolism is essential for activation of human platelets ex vivo as well as thrombus formation in a murine model in vivo. Considering that a substantial body of information on the safety and pharmacokinetics of DCA and DHEA in humans is already available, ${ }^{8-11}$ one can expect probable future translation of these drugs into clinical use as antiplatelet agents.

\section{Methods}

\section{Ethical approval}

The animal experiments were approved by the Central Animal Ethical Committee of the Institute of Medical Sciences, Banaras Hindu University. All efforts were made to minimize the number of animals used, and their suffering. Peripheral venous blood samples were collected from healthy participants after obtaining written informed consent, strictly in accordance with the recommendations and as approved by the ethical committee of the Institute of Medical Sciences, Banaras Hindu University.

\section{Platelet preparation and mitochondrial respirometry}

Platelets were isolated from fresh human blood by differential centrifugation, as already described. ${ }^{12}$ Mitochondrial respiration was measured using a high-resolution respirometer (Oxygraph$2 \mathrm{k}$, Oroboros Instruments) as previously described. ${ }^{12}$ Details are provided in the Online Supplement.

\section{Measurements of glucose, lactate and NAPDH}

Rates of glucose uptake and lactate secretion in thrombin $(0.5$ $\mathrm{U} / \mathrm{mL}$ )-stimulated platelets were determined using an YSI 2900D Multiparameter Bioanalytical System (YSI Life Sciences), which uses immobilized enzyme electrodes and electrochemistry-based biosensing. ${ }^{13}$

The ratio of $\mathrm{NADPH}$ to total $\mathrm{NADP}(\mathrm{H})$ in washed human platelets was determined using a NADP/NADPH assay kit (Sigma). For this, NADP was decomposed at $60^{\circ} \mathrm{C}$ for $30 \mathrm{~min}$ followed by estimation of the amount of NADPH. To estimate total $\operatorname{NADP}(\mathrm{H})$, extract was directly processed for color development following the instructions of the kit's manufacturer and absorbance was recorded at $450 \mathrm{~nm}$.

\section{Platelet aggregation and granule secretion}

Platelets suspended in buffer B or in platelet-rich plasma were stirred $(1200 \mathrm{rpm})$ at $37^{\circ} \mathrm{C}$ in an optical lumi-aggregometer (Chrono-log model 700-2) for $1 \mathrm{~min}$. Platelet aggregation induced with thrombin or collagen was recorded as percent change in light transmittance where $100 \%$ reflects transmittance through a blank (buffer or platelet-poor plasma). ${ }^{12}$

Release of adenine nucleotides from platelet dense granules was measured with the Chronolume reagent (stock concentration, 0.2 $\mu \mathrm{M}$ luciferase/luciferin). Luminescence generated was monitored using the lumi-aggregometer in parallel with the platelet aggregation measurement. ${ }^{12}$

Secretion from platelet $\alpha$-granules was evaluated by quantifying surface expression of P-selectin as described previously. ${ }^{12}$

Details are provided in the Online Supplement.

\section{Platelet surface integrin activation and GLUT3 expression}

Platelet surface integrin activation was analyzed as described previously. ${ }^{12}$

In order to measure GLUT3 surface expression, platelets were fixed with an equal volume of $4 \%$ paraformaldehyde, washed and blocked with $2 \%$ bovine serum albumin for $40 \mathrm{~min}$ before being incubated with a GLUT3-specific antibody for $2 \mathrm{~h}$, and then washed and stained with Alexa Fluor 488-conjugated goat antimouse antibody for $1 \mathrm{~h}$. Cells were analyzed by flow cytometry as described above.

Further details are provided in the Online Supplement.

\section{Measurement of intracellular reactive oxygen species}

The intracellular levels of reactive oxygen species (ROS) were determined using a redox-sensitive cell-permeable dye, $\mathrm{H}_{2} \mathrm{DCF}$ DA as described elsewhere. ${ }^{14}$ Details are available in the Online Supplement.

\section{Immunoblotting}

Immunoblotting experiments were performed as described previously. ${ }^{12}$ Details are given in the Online Supplement.

\section{Studies of coagulant activity}

Externalization of phosphatidylserine, a measure of surface procoagulant activity, in stimulated platelets was assessed from annexin $\mathrm{V}$ binding, as described previously. ${ }^{15}$

\section{Animal experiments}

Intravital microscopy of ferric chloride-induced thrombosis of mice mesenteric arterioles was performed as described elsewhere ${ }^{16}$ with some modifications.

Tail bleeding time experiments were carried out as described previously, ${ }^{17}$ with minor modifications.

Pulmonary embolism was induced by collagen-epinephrine in 12- to 20 -week old Swiss albino mice of either sex as described previously. $^{12}$

The methods are described in detail in the Online Supplement.

\section{Results}

\section{Platelets exhibit a higher rate of aerobic glycolysis upon agonist stimulation}

We measured oxygen flux in platelets (suspended in Tyrode modified buffer under stirring) using a Clark amperometric electrode at high resolution (sampling at $2 \mathrm{~s}$ intervals). This system in a cuvette format closely resembles the platelet aggregometry of Born. When we exposed 
platelets to thrombin $(0.5 \mathrm{U} / \mathrm{mL})$, a strong physiological agonist, there was a rapid surge in oxygen consumption rate over the basal respiration (within the first $90 \mathrm{~s}$ of stimulation) (Figure 1A,B), apparently driven by energydemanding early platelet responses. This upsurge in oxygen consumption rate was, however, followed by an abrupt fall (Figure 1A,B) that, intriguingly, coincided with the exponentially rising phase of the platelet aggregatory response (Online Supplementary Figure S1A). We recorded identical peaking and plunging of oxygen consumption in platelets upon stimulation with collagen, another physiological agonist (Online Supplementary Figure S1B).

We sought to identify the reason underlying this rapid plunge in oxygen consumption of stimulated platelets, despite continued ATP requirement for energy-intensive processes such as cytoskeletal reorganization, shedding of extracellular vesicles, and protein synthesis, which are all associated with platelet activation. As aggregate formation could restrict access of oxygen to cells persisting within the core of the aggregate mass, we performed respirometry experiments after pre-treatment with ArgGly-Asp-Ser (RGDS), a tetrapeptide that prevents platelet aggregation. Strikingly, we noticed no difference in polarogram profiles (Online Supplementary Figure S1C), which ruled out any contribution of cell-cell aggregate formation to the observed drop in oxygen consumption. A reduced rate of cellular respiration could reflect dysfunctional mitochondria in agonist-treated platelets. ${ }^{18} \mathrm{We}$ found that oxygen flux following sequential treatment of platelets with oligomycin (an inhibitor of ATPase), carbonyl cyanide $\mathrm{m}$-chlorophenyl hydrazine (an uncoupler), and antimycin A (an inhibitor of respiratory complex III) was consistent with the presence of well-coupled and viable mitochondria in stimulated platelets (Online Supplementary Figure S2A). This prompted us to hypothesize a Warburg-like phenomenon ${ }^{19}$ in stimulated platelets. Consistent with this proposition, the decline in oxygen flux was obviated when platelets were exposed to DCA (Figure 1A,B), which enhances flux through the tricarboxylic acid (TCA) cycle by promoting pyruvate dehydrogenase activity, or to methylene blue, an alternative electron carrier (Online Supplementary Figure S2B).

The Warburg effect, or aerobic glycolysis, entails augmented uptake of glucose from external medium by the cells. We detected 1.8- and 2-fold increases in glucose uptake and lactate generation, respectively, by platelets upon exposure to thrombin (Figure 1C). Enhanced glucose uptake by stimulated platelets is mediated through cell membrane translocation of cytosolic preformed GLUT3. ${ }^{20}$ As expected, we observed a nearly $41 \%$ rise in surface expression of GLUT3 upon stimulation of platelets with thrombin $(0.5 \mathrm{U} / \mathrm{mL})$ (Figure $1 \mathrm{D}, \mathrm{E})$. The surface mobilization of GLUT3 in neuronal cells is regulated by the activity of AMP-activated protein kinase (AMPK). ${ }^{21}$ In line with this, pre-treatment of platelets with compound C, which is a specific inhibitor of AMPK, resulted in a significant drop (by $31 \%$ ) of GLUT3 expression on thrombin-stimulated platelets (Figure 1D,E).

\section{Stimulated platelets switch to aerobic glycolysis through negative regulation of pyruvate dehydrogenase and pyruvate kinase M2}

We next sought to elucidate the mechanism underlying the observed switch to aerobic glycolysis from oxidative phosphorylation in stimulated platelets. Pyruvate dehy- drogenase $(\mathrm{PDH})$, the 'gate-keeper' enzyme, determines the relative fluxes through glycolysis and oxidative phosphorylation. PDH activity is regulated through inhibitory phosphorylation (at S293) by pyruvate dehydrogenase kinase (PDK). ${ }^{22}$ We examined the expression of phosphorylated PDH in thrombin-stimulated platelets by using a phospho-specific antibody. Thrombin $(0.5 \mathrm{U} / \mathrm{mL})$ induced a rise in the level of phosphorylated $\mathrm{PDH}$ in platelets (Figure 2A,B), which diverts flux away from the TCA cycle. DCA $(20 \mathrm{mM})$, a pharmacological inhibitor of PDK, almost completely abolished phosphorylation of $\mathrm{PDH}$ (Figure $2 \mathrm{~A}, \mathrm{~B}$ ) and partially restored the drop in oxygen consumption in thrombin-treated platelets (Figure 1A lower panel, 1B). Pre-exposure to DCA also led to significant inhibition of the thrombin-induced rises in the rates of glucose uptake and lactate generation (by 33\% and $28 \%$, respectively) (Figure 1C), suggesting PDK-mediated inactivation of $\mathrm{PDH}$ and decreased flux through the TCA cycle in stimulated platelets. Interestingly, DCA also triggered a 20\% drop in GLUT3 externalization (Figure 1D,E) in thrombin-stimulated platelets. AMPK is known to induce phosphorylation of PDH under conditions of nutrient-deprivation leading to inhibition of PDH activity. ${ }^{23}$ Exposure to compound $\mathrm{C}$ reversed the increases in $\mathrm{PDH}$ phosphorylation (Figure 2A,B) as well as glucose uptake/lactate secretion rates (Figure $2 \mathrm{E}$ ) that were observed in thrombin-stimulated platelets. As AMPK activity in platelets is upregulated by thrombin, ${ }^{24}$ this kinase is positioned in the thrombin signaling pathway, upstream of $\mathrm{PDH}$.

Neoplastic transformation of cells is associated with expression of PKM2, a splice variant of pyruvate kinase, which, unlike PKM1, facilitates aerobic glycolysis through a low-activity dimer state. ${ }^{25}$ We report that PKM2 is significantly expressed in human platelets (Online Supplementary Figure S3A). Expression of the PKM2 mRNA splice form was remarkably higher than that of its PKM1 counterpart (Online Supplementary Figure S3B). Phosphorylation of PKM2 at Y105 is associated with sustenance of the dimer state with attenuated catalytic activity. ${ }^{26}$ Using a phosphospecific antibody we observed that exposure to thrombin $(0.5 \mathrm{U} / \mathrm{mL})$ evoked appreciably higher phosphorylation (Y105) of PKM2, suggesting low enzymatic activity in stimulated platelets. This phosphorylation was reversed by PP2, an inhibitor of Src family tyrosine kinases, which are activated in stimulated platelets (Figure 2C). PP2 also regressed the thrombin-induced increases in glucose uptake/lactate secretion rates in platelets (Figure 2E). Pretreatment of cells with DASA $(200 \mu \mathrm{M})$, an activator of PKM2, decreased thrombin-induced GLUT3 externalization by $36 \%$ (Figure $1 \mathrm{D}, \mathrm{E})$. Thus, our findings suggest that stimulated platelets make a metabolic switch to aerobic glycolysis through post-translational regulation of $\mathrm{PDH}$ and PKM2 enzyme activities.

\section{Enhanced flux through the pentose phosphate pathway supports reactive oxygen species-dependent integrin activation}

Aerobic glycolysis and low PKM2 activity would lead to pooling of glycolytic intermediates upstream of pyruvate, which include glucose-6-phosphate, a substrate for the PPP. ${ }^{25}$ Hence, we studied metabolic flux through the PPP in thrombin-stimulated platelets, as reflected by the ratio of NADPH to total NADP(H) levels. Thrombin $(0.5 \mathrm{U} / \mathrm{mL})$ induced a significant rise (by $50 \%$ ) in the ratio of $\mathrm{NADPH}$ 

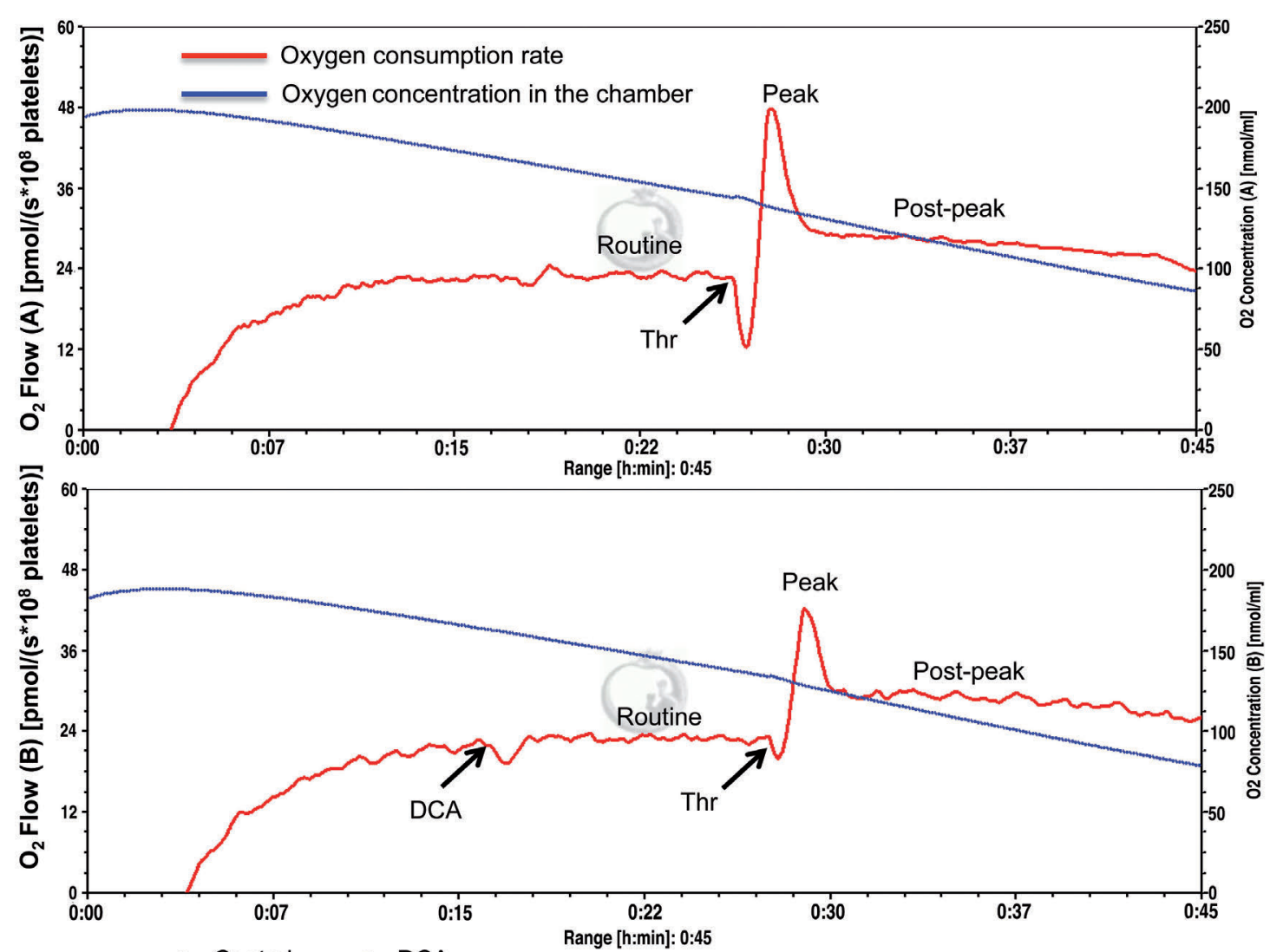

B

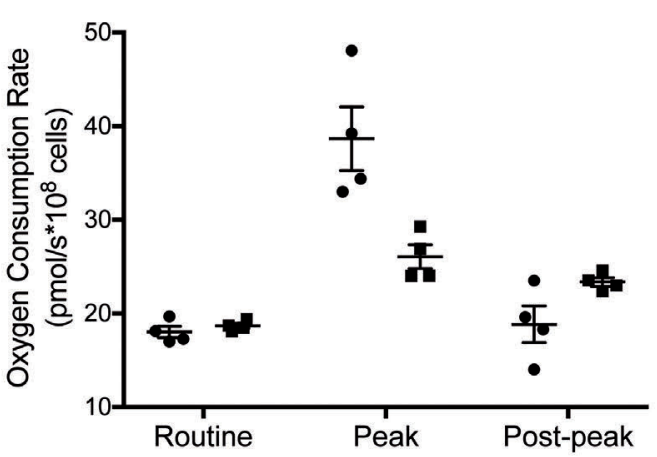

D

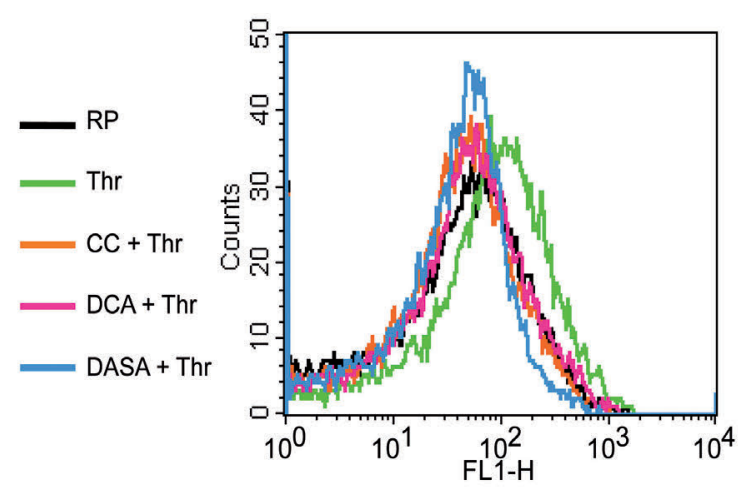

C

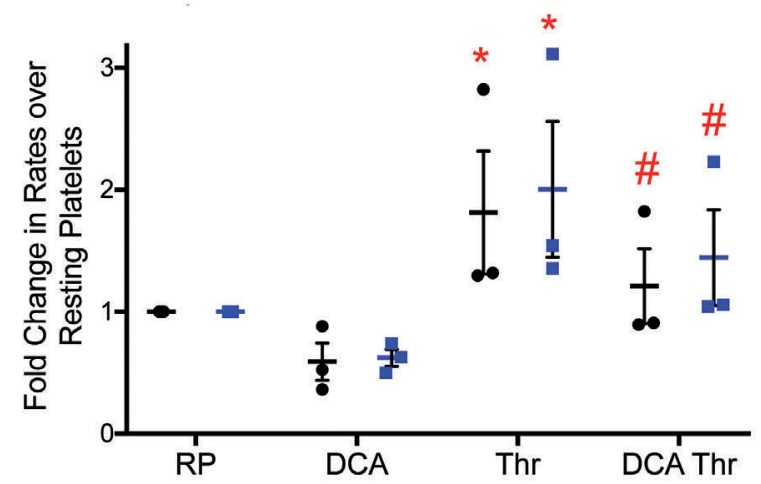

E

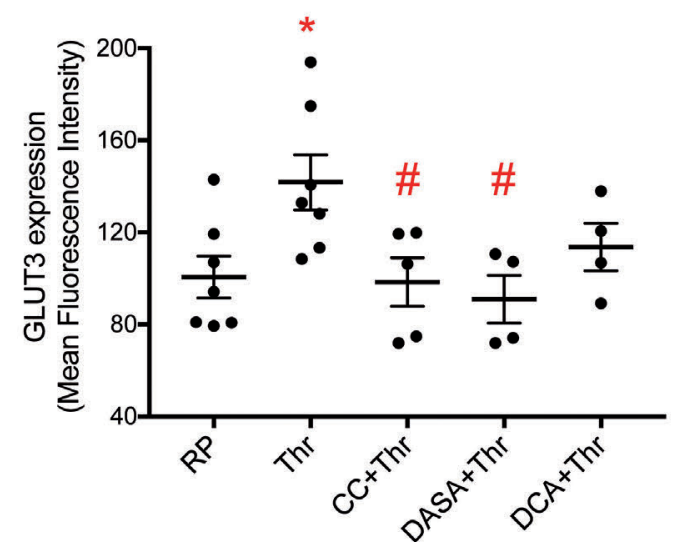

Figure 1. Platelets exhibit higher rate of aerobic glycolysis upon agonist stimulation. (A) Polarograms exhibiting oxygen flow or oxygen concentration in thrombin (0.5 $\mathrm{U} / \mathrm{mL}$ )-stimulated platelets suspended in Tyrode modified buffer. The platelets were not pretreated (upper panel) or were pretreated with DCA (20 mM) (lower panel). The blue line represents oxygen concentration within the chamber while the red line traces the rate of oxygen consumption by the cells. (B) Scatter dot plots representing routine, peak (following thrombin addition) and post-peak respiration in platelets. (C) Fold change in the rate of glucose uptake (black circles) and lactate secretion (blue squares) in platelets treated with different reagents as indicated. (D and E) GLUT3 externalization as observed by flow cytometry in platelets treated with different reagents as indicated. Data are presented as the mean \pm standard error of mean. Each dot represents an independent observation. $(* P<0.05$ as compared to resting platelets; $\# P<0.05$ as compared to thrombin-stimulated platelets). CC, compound C; DCA: dichloroacetate; RP: resting platelets; Thr: thrombin. 
A

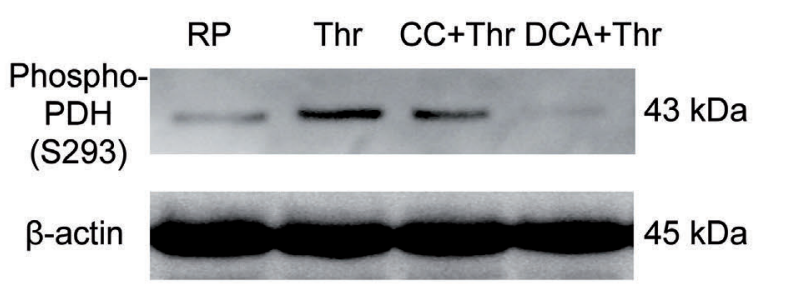

C

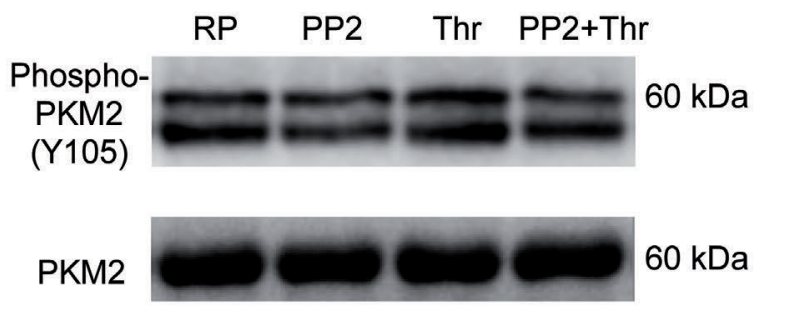

E

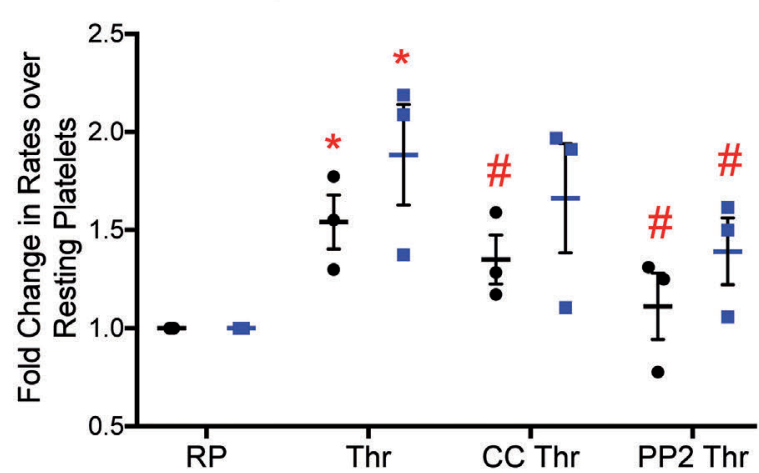

G

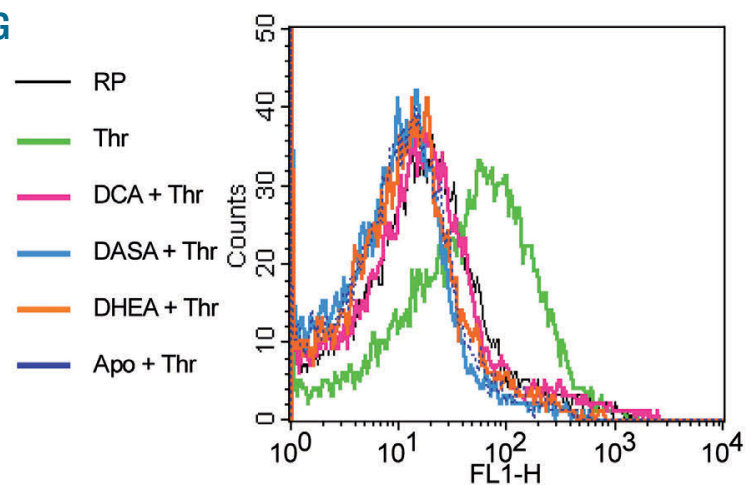

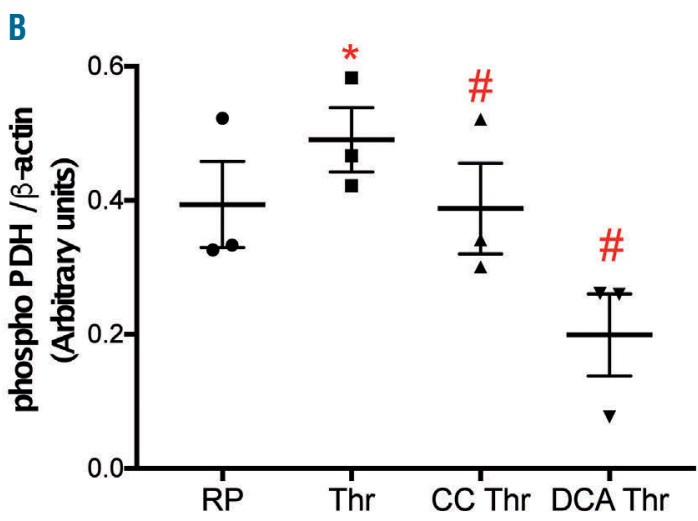

D

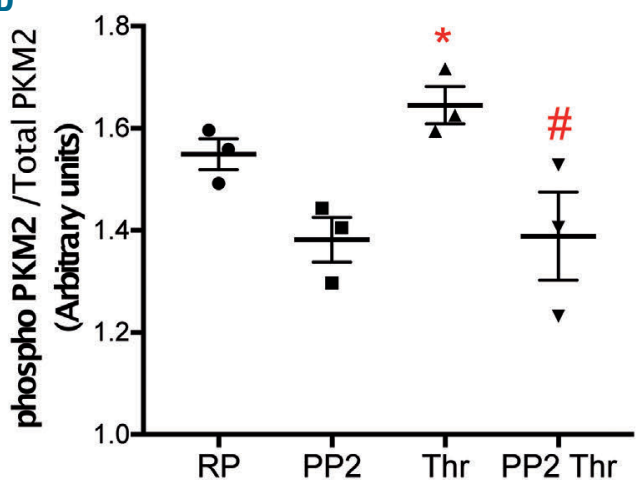

F
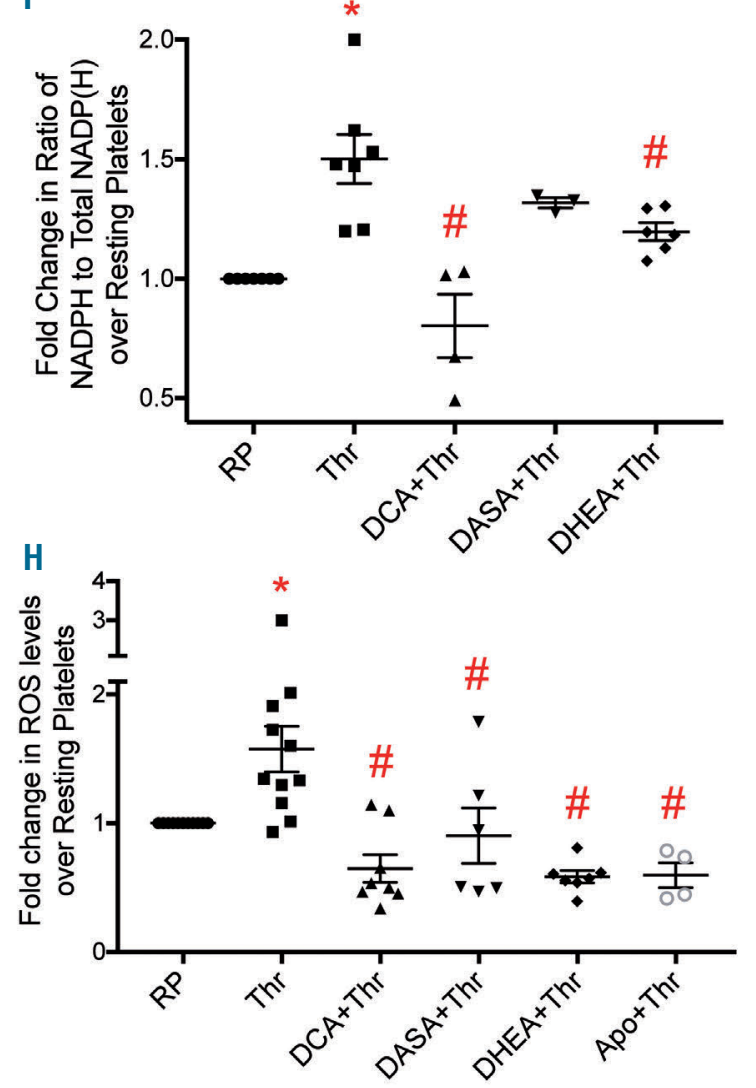

Figure 2. Regulation of aerobic glycolysis and the pentose phosphate pathway in stimulated platelets. (A and C) Representative immunoblots showing expression of phospho-PDH (Ser 293) and phospho-PKM2 (Tyr 105), respectively, in platelets treated with different reagents (thrombin, $0.5 \mathrm{U} / \mathrm{mL}$; compound C, 50 uM; DCA, $20 \mathrm{mM}$; PP2, $50 \mu \mathrm{M})$ as indicated. (B and D) Corresponding scatter dot plots showing expression of phospho-PDH relative to $\beta$-actin and phospho-PKM2 relative to total PKM2, respectively, after densitometry analysis. (E) Glucose uptake and lactate secretion rates in platelets treated with different reagents. (F) Scatter dot plot showing the ratio of NADPH to total $\mathrm{NADP}(\mathrm{H})$ in platelets treated with different reagents as indicated. ( $\mathrm{G}$ and $\mathrm{H}$ ) ROS generation as detected by H2DCFDA dye in platelets treated with different reagents as indicated. The dose of apocynin was $600 \mu \mathrm{M}$. Data are presented as the mean \pm standard error of mean. Each dot represents an independent observation. (*P<0.05 as compared to resting platelets; ${ }^{~} P<0.05$ as compared to thrombin-stimulated platelets). Apo: apocynin; CC, compound C; DCA: dichloroacetate; DASA: diarylsulfonamide; DHEA: dehydroepiandrosterone sulphate; PDH: pyruvate dehydrogenase; PKM2: pyruvate kinase splice variant; RP: resting platelets; Thr: thrombin. 
to total $\operatorname{NADP}(\mathrm{H})$, which was reversed by up to $20 \%$ in the presence of DHEA $(200 \mu \mathrm{M})$ (Figure 2F). DHEA, an endogenous steroid hormone, is an inhibitor of glucose 6phosphate dehydrogenase, the rate-limiting enzyme of the PPP. Small-molecule modulators that facilitate the TCA cycle and check the rate of aerobic glycolysis would also restrict flux of metabolites through the PPP. Predictably, pre-exposure of platelets to DCA $(20 \mathrm{mM})$ or DASA $(200 \mu \mathrm{M})$ led to reductions in the ratio of NADPH to total $\mathrm{NADP}(\mathrm{H})$ of $47 \%$ and $12 \%$, respectively (Figure 2F).

Activity of NADPH oxidase (NOX) has been widely documented as a significant source of ROS in stimulated platelets. ${ }^{27,28}$ We investigated whether NADPH accumulated in thrombin-stimulated platelets acts as a substrate for $\mathrm{NOX}$, and leads to enhanced generation of ROS. In keeping with this possibility, pre-incubation of platelets with either DHEA or apocynin (a NOX inhibitor) prior to exposure to thrombin resulted in $63 \%$ and $62 \%$ attenuation in ROS generation, respectively. Remarkably, pre-treatment with DCA and DASA also led to $59 \%$ and $43 \%$ decreases, respectively, in ROS production in thrombin-stimulated platelets (Figure 2G,H). These decreases were consistent with the impeded PPP flux observed in the presence of these molecules.

There is now considerable evidence to suggest that ROS are important mediators of the platelet activation signaling $^{29}$ that culminates in the expression of conformationally-active integrin $\alpha_{\text {II }} \beta_{\text {III }}{ }^{27}$ Consistently, DHEA as well as NOX inhibitors - DPI and apocynin - attenuated the binding of PAC-1 (an antibody directed against conformationally-active integrin $\alpha_{\mathrm{II}} \beta_{\mathrm{III}}$ ) to thrombin-stimulated platelets by $59 \%, 56 \%$, and $62 \%$, respectively (Figure $3 \mathrm{~A}, \mathrm{~B})$. DCA and DASA, which limit flux through the PPP, also inhibited PAC-1 binding by $32 \%$ and $65 \%$, respectively. Furthermore, the role of ROS in mediating platelet activation was underscored by near-total abrogation of thrombin-induced PAC-1 binding to platelets in the presence of $\mathrm{N}$-acetyl cysteine $(10 \mathrm{mM})$, a ROS scavenger (Online Supplementary Figure S4). As an active conformer of $\alpha_{\mathrm{II}} \beta_{3}$ is endowed with high affinity towards fibrinogen, we next studied the association of fluorescently labeled fibrinogen to thrombin-stimulated platelets in the presence of these small-molecule modulators. DCA, DASA, DHEA, DPI and apocynin attenuated fibrinogen binding to stimulated platelets by $28 \%, 58 \%, 37 \%, 49 \%$, and $63 \%$ respectively (Figure $3 \mathrm{C}, \mathrm{D})$.

\section{Small-molecule inhibitors of aerobic glycolysis and the pentose phosphate pathway impair platelet responsiveness}

As we demonstrated that small-molecule inhibitors of aerobic glycolysis and the PPP could prevent platelet surface integrins $\alpha_{\mathrm{II}} \beta_{3}$ from switching to an active conformation and attenuate fibrinogen binding, we asked whether they could also inhibit activation-initiated platelet responses including aggregation and secretion of granule contents. Pre-treatment with DCA (20 mM), DASA (200 $\mu \mathrm{M})$ or DHEA $(200 \mu \mathrm{M})$ led to reductions in platelet aggregation evoked by thrombin $(0.5 \mathrm{U} / \mathrm{mL})$ by $61 \%, 58 \%$ and $19 \%$, respectively (Figure 3E,F). A similar inhibition of collagen $(2 \mu \mathrm{g} / \mathrm{mL})$-induced platelet aggregation, albeit to a much greater extent, was also observed (Figure $3 \mathrm{G}, \mathrm{H}$ ), which was consistent with a previous report that described inhibition of platelet aggregation by DHEA in an Akt/ERK/p38 MAPK-dependent fashion. ${ }^{30}$ Surface externalization of $\mathrm{P}$-selectin from $\alpha$-granules and secretion of adenine nucleotides stored in dense granules dropped upon pre-treatment with DCA, DASA or DHEA (Online Supplementary Figure S5). These small molecules also inhibited phosphatidylserine exposure (measured through annexin $\mathrm{V}$ binding) on the surface of stimulated platelets (Figure 3I,J), which is critical for the procoagulant activity of the platelets. ${ }^{31}$ Thrombin elicited shedding of extracellular vesicles (size range, $25-800 \mathrm{~nm}$; predominantly 100 $250 \mathrm{~nm}$ ) bearing a phosphatidylserine-rich procoagulant surface $^{32}$ from platelets, a phenomenon which was also mitigated by $\sim 40 \%$ in the presence of DCA (Online Supplementary Figure S6). The foregoing observations strongly underscore the significance of aerobic glycolysis and the PPP-NOX axis in platelet activation and thrombosis. In contrast to DCA, pre-treatment with either antimycin or oligomycin did not significantly inhibit platelet aggregation induced by thrombin $(0.2 \mathrm{U} / \mathrm{mL})$ (Online Supplementary Figure S7), suggesting that mitochondrial respiration is relatively dispensable for platelet activation responses. To rule out the possibility that the observed attenuation in platelet activity was a consequence of cell death induced by the small-molecule modulators, we performed a lactate dehydrogenase leak assay. There was no significant release of lactate dehydrogenase activity from platelets when cells were exposed to DCA $(20 \mathrm{mM})$, DASA $(200 \mu \mathrm{M})$ or DHEA $(200 \mu \mathrm{M})$ for up to 2 h (Online Supplementary Figure S8).

\section{Small-molecule inhibitors of aerobic glycolysis and the pentose phosphate pathway preclude thrombosis in mice}

Platelets play a pivotal role in the pathogenesis of arterial thrombosis which underlies occlusive vasculopathies such as acute myocardial infarction and ischemic stroke. In order to establish a link between energy metabolism in activated platelets and arterial thrombosis in vivo, we studied the effect of small-molecule metabolic modulators in a murine model of mesenteric thrombosis induced by ferric chloride. We fluorescently labeled the platelets and induced intramural thrombus in exteriorized mesenteric arterioles of mice administered DCA (200 mg/kg intraperitoneal), DHEA (50 $\mathrm{mg} / \mathrm{kg}$ intraperitoneal), DASA (40 $\mathrm{mg} / \mathrm{kg}$ intravenous) or vehicle (control). Intravital imaging of thrombus formation was carried out by epifluorescence video microscopy using a high-speed camera. We documented the time required for initial thrombus formation, rate of thrombus growth and time to occlusion as pointers to the initiation, propagation and stabilization of thrombus, respectively. Strikingly, mice pre-treated with DCA (Online Supplementary Video S2), DHEA (Online Supplementary Video S3) or DASA (Online Supplementary Video 54 ) had significantly prolonged mean times to form first thrombus compared to vehicle-treated mice (DCA, $9.6 \pm 1.2 \mathrm{~min}$; DHEA, $11.8 \pm 1.02 \mathrm{~min}$; DASA, $9.67 \pm 1.2$ min, versus control, $5.9 \pm 0.60 \mathrm{~min}$ ) (Figure 4A,B) and impaired thrombus growth rate (Figure 4A,C,D) compared to vehicle-treated mice (Online Supplementary Video S1). While the mean occlusion time for control mice was $20.4 \pm 2.38 \mathrm{~min}$, stable occlusion failed to occur even after $40 \mathrm{~min}$ from the time of injury in all DHEA-pretreated mice and most of the mice administered DCA or DASA (except one from each group) (Figure 4E). These results suggest that agents that block metabolic reprogramming 
A

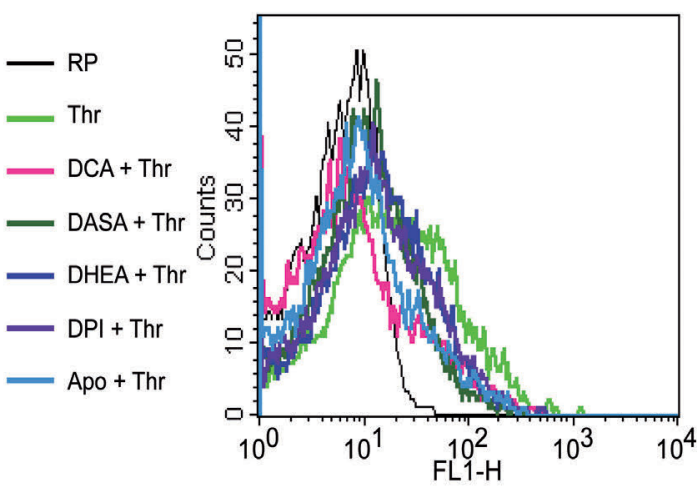

C

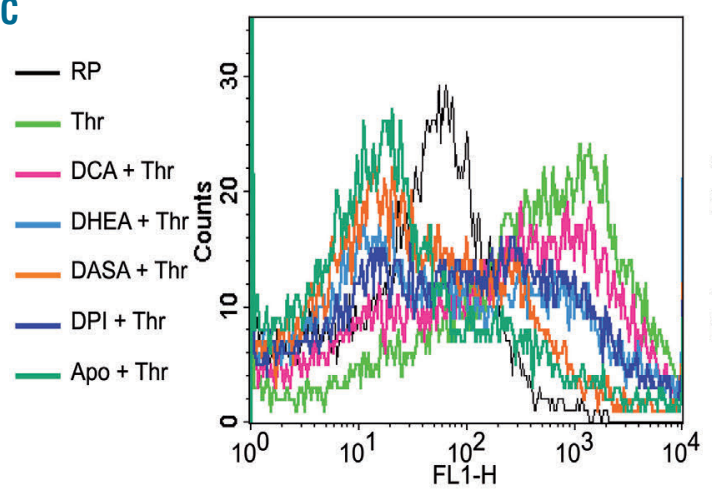

E

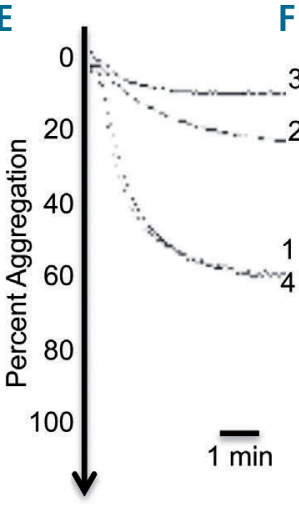

F

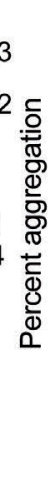

G

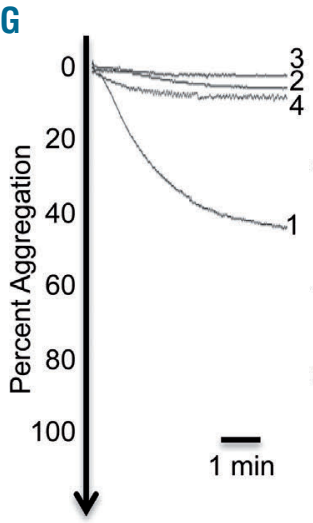

$\mathrm{H}$
B

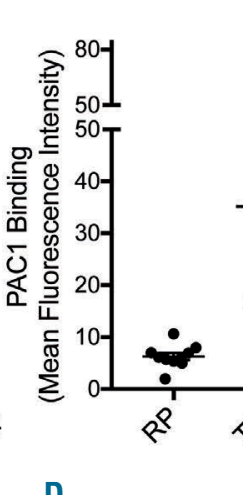

D
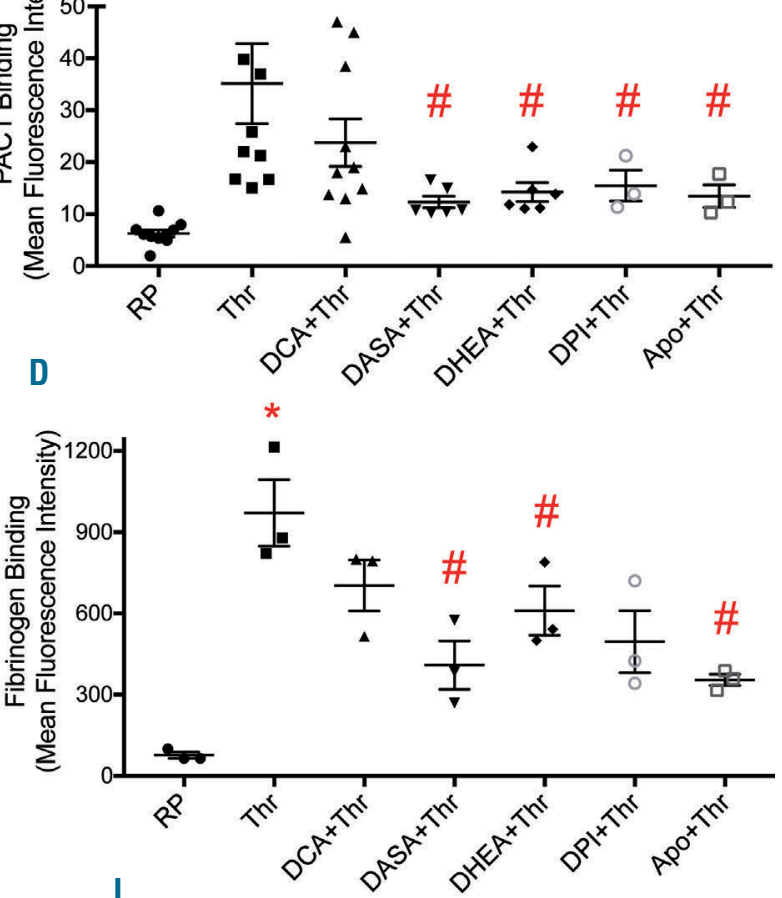

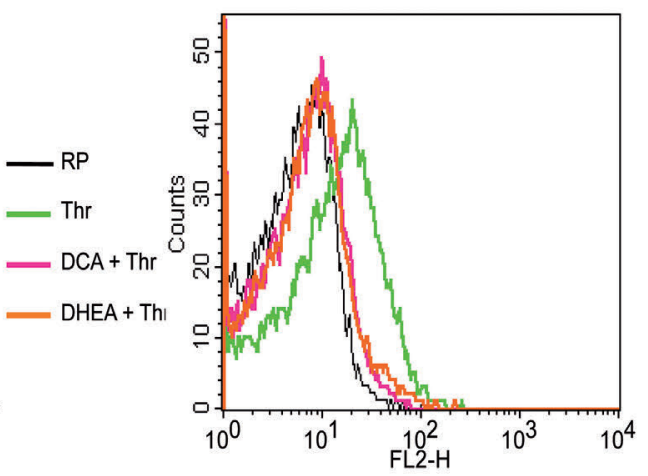

J
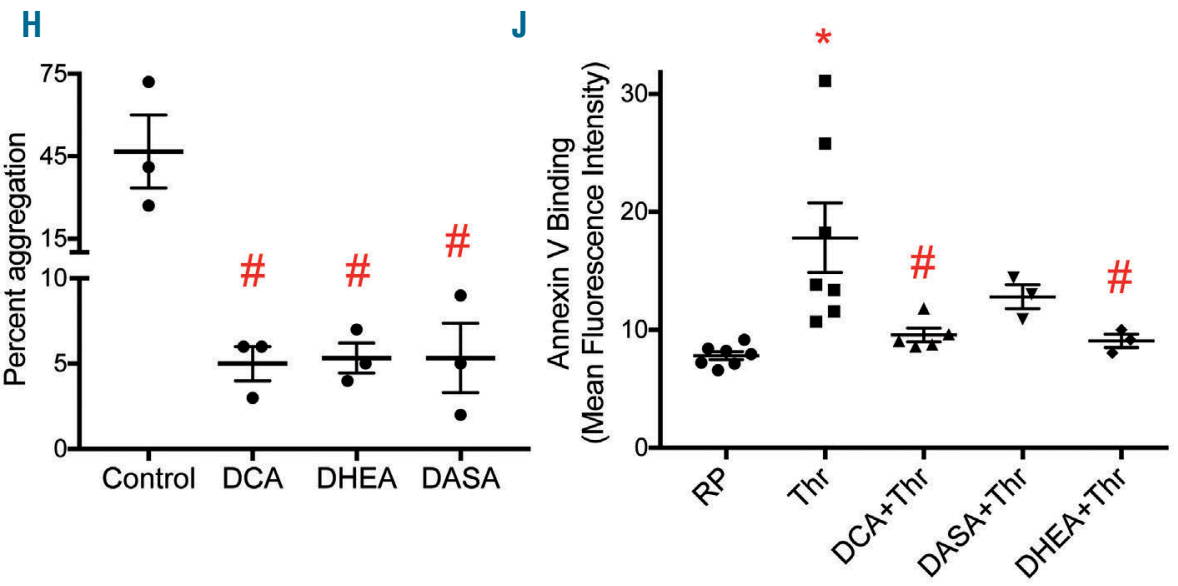

Figure 3. Decreased metabolic flux through the pentose phosphate pathway impairs platelet responsiveness to agonist stimulation. (A and B) PAC-1 binding in platelets treated with different reagents as indicated. (C and D) Binding of fluorescent fibrinogen to platelets treated with different reagents as indicated. (E and $\mathrm{G}$ ) Platelet aggregation induced by thrombin $(0.2 \mathrm{U} / \mathrm{mL})$ and collagen $(2 \mu \mathrm{g} / \mathrm{mL})$, respectively in the presence of vehicle (control), DCA $(20 \mathrm{mM})$, DASA $(200 \mu \mathrm{M})$ or DHEA $(200 \mu \mathrm{M})$ (tracings $1,2,3$ and 4 respectively). ( $F$ and $H$ ) Scatter dot plots showing platelet aggregation. (I and J) Fluorescent-labeled annexin $V$ binding to platelets treated with various reagents as indicated. Data are presented as the mean \pm standard error of mean. Each dot represents an independent observation. ( ${ }^{*}<<0.05$ as compared to resting platelets; ${ }^{\# P}<0.05$ as compared to thrombin-stimulated platelets). Apo: apocynin; DASA: diarylsulfonamide; DCA: dichloroacetate; DHEA: dehydroepiandrosterone sulphate; RP: resting platelets; Thr: thrombin 
to aerobic glycolysis and the PPP in stimulated platelets cause profound impairment of arterial thrombosis in vivo.

We corroborated the above findings in a mouse model of collagen-epinephrine-induced pulmonary embolism. Mice were pretreated with DCA (200 mg/kg), DHEA (50 $\mathrm{mg} / \mathrm{kg}$ ), DASA (40 mg/kg) or vehicle (control), followed by intravenous administration of a collagen-epinephrine mixture to induce pulmonary embolism. Hematoxylin \& eosin-stained lung sections from mice pretreated with small-molecule inhibitors displayed significantly fewer thrombosed pulmonary vessels [DCA, $4.12 \pm 1.4$ per low power field (lpf); DHEA, $4.02 \pm 0.82 / \mathrm{lpf}$; DASA, $3.03 \pm$
A

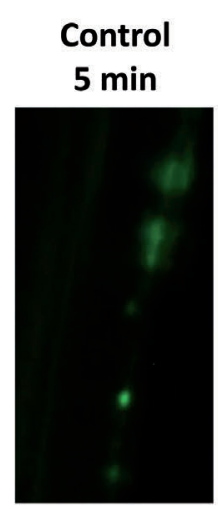

DCA 5 min
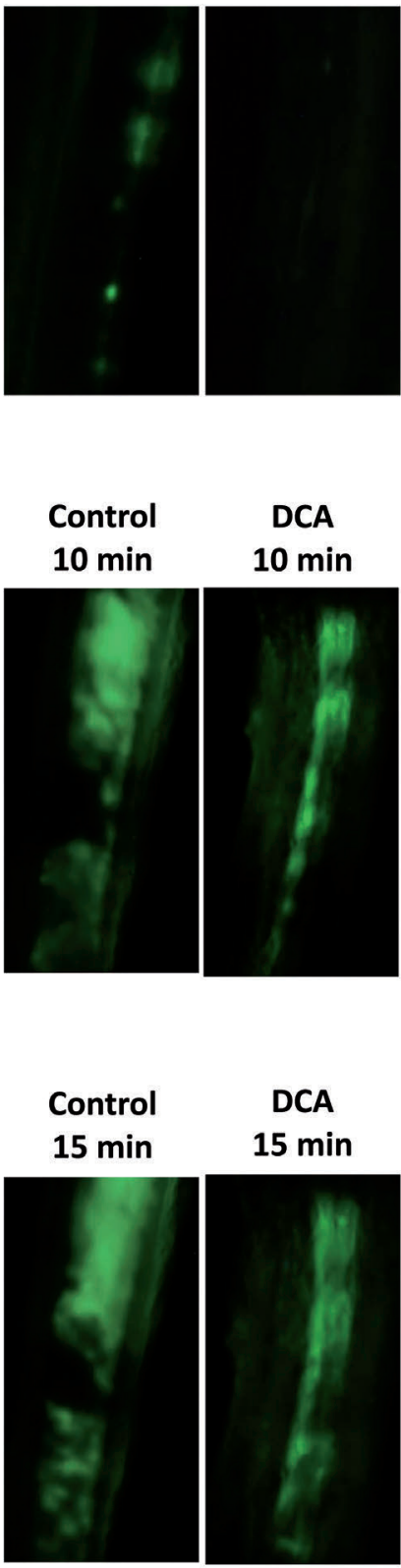

\begin{abstract}
DCA
\end{abstract} $15 \mathrm{~min}$

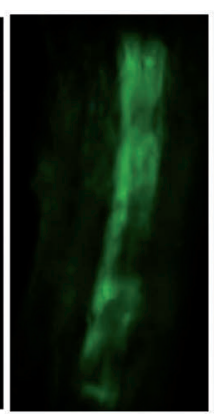

DHEA $10 \mathrm{~min}$

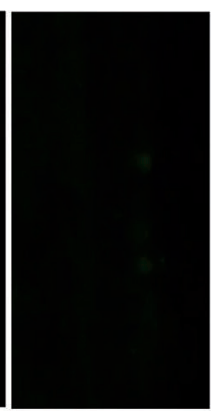

DHEA $15 \mathrm{~min}$

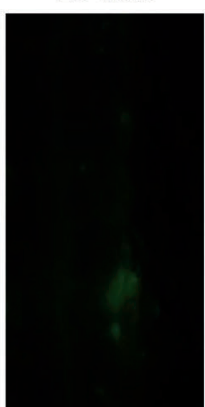

DASA

$10 \mathrm{~min}$

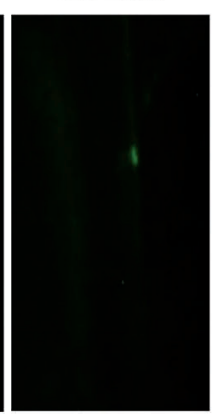

DASA $15 \mathrm{~min}$

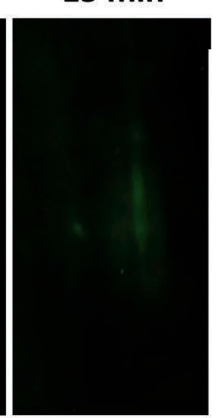

B $*$
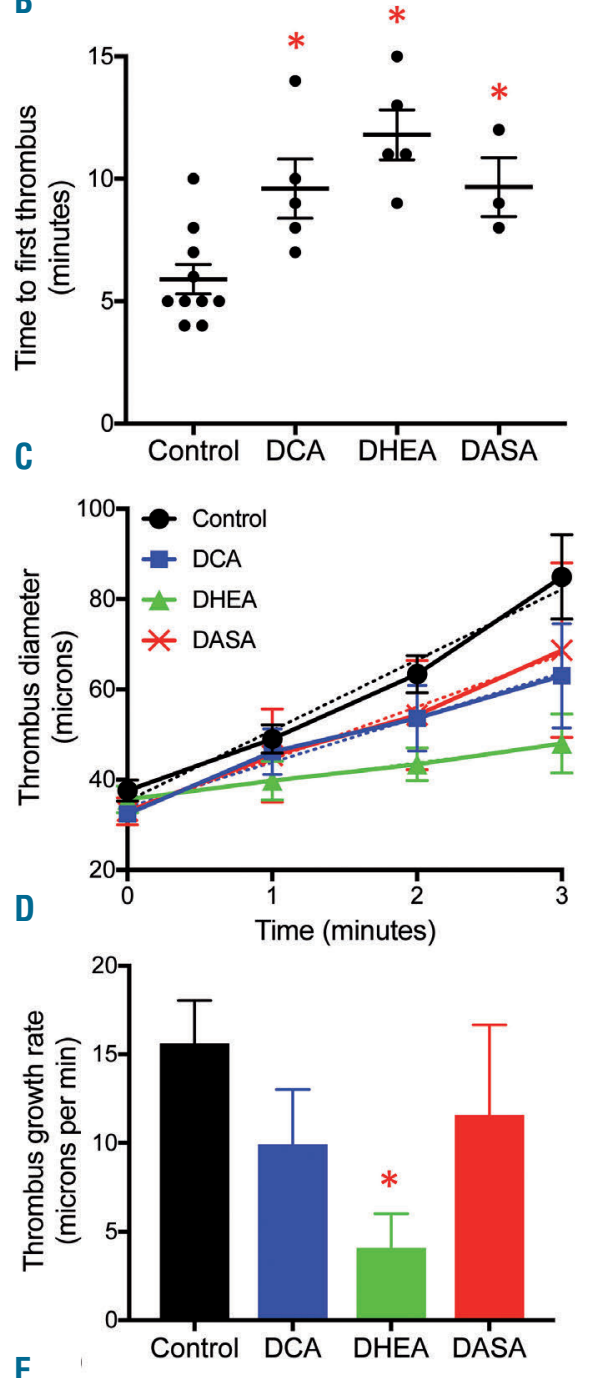

E

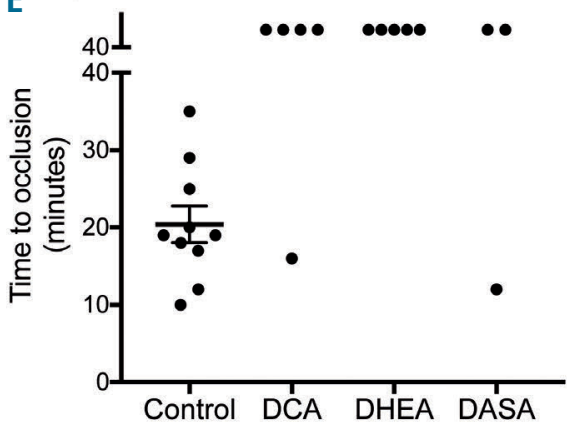

Figure 4. Small-molecule inhibitors of aerobic glycolysis and the pentose phosphate pathway impair thrombus formation in mice. (A) Representative time lapse images exhibiting thrombus formation in mice, pre-administered either vehicle (control), DCA, DHEA, or DASA captured 5, 10 or 15 min after injury of mesenteric arterioles of $>100 \mu \mathrm{m}$ diameter with ferric chloride. (B and E) Scatter dot plots representing, respectively, time to first thrombus formation and time to stable occlusion in mice pre-administered vehicle (control), DCA, DHEA or DASA. (C and D) Line graph and bar diagram showing thrombus growth rate in different treatment groups, as indicated. Each dot in the scatter plots represents an independent observation. Data are expressed as the mean \pm standard error of mean. $* P<0.05$ with respect to vehicle-treated mice. DASA: diarylsulfonamide; DCA: dichloroacetate; DHEA: dehydroepiandrosterone sulphate. 
0.74 /lpf; versus control $8.1 \pm 0.95 / \mathrm{lpf}$ ) with erythrocyte extravasation in some fields. These observations establish that prohibition of platelet aerobic glycolysis or the PPP prevents thrombosis, and may impair hemostasis.

We next evaluated the effect of metabolic modulators on primary hemostasis in mice by a tail-bleeding assay. Administration of DCA (200 mg/ $\mathrm{kg}$, intraperitoneal), DHEA $(50 \mathrm{mg} / \mathrm{kg}$, intraperitoneal) or DASA (40 mg/kg, intravenous) to the mice was associated with prolonged bleeding times (Figure 5C) as well as an increase in the amount of bleeding compared to that of vehicle-treated animals (Online Supplementary Figure S9). These observations establish that aerobic glycolysis and consequent flux through the PPP are also essential for hemostasis.

\section{Discussion}

The essence of platelet function is response to stimuli. Platelets respond to hemostatic cues with a series of activ-
A

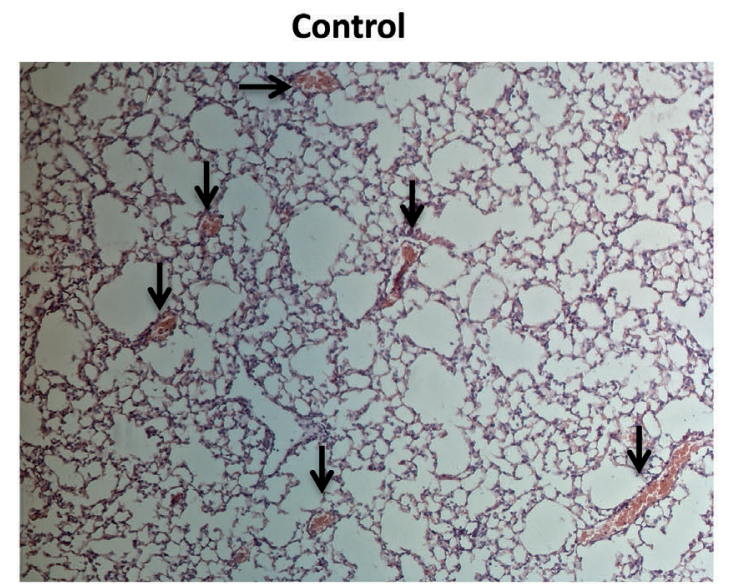

DHEA

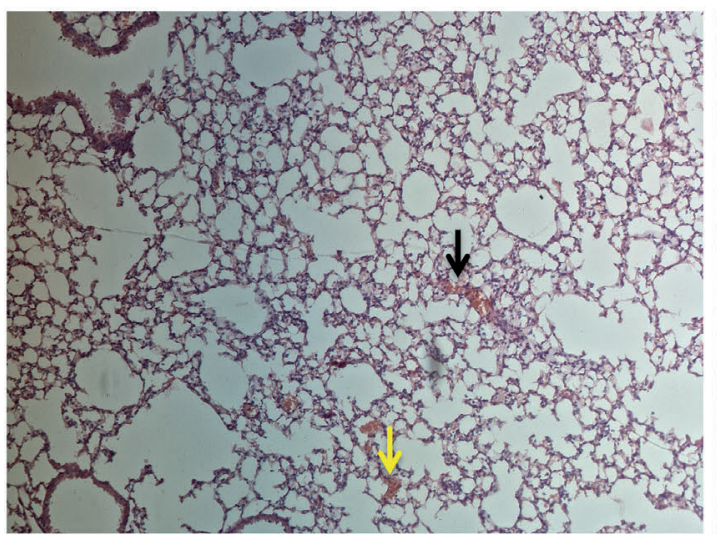

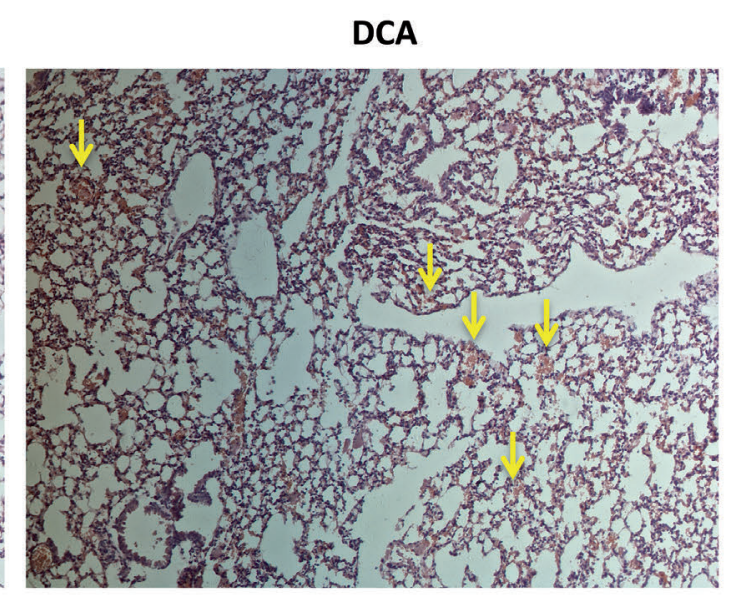

DASA
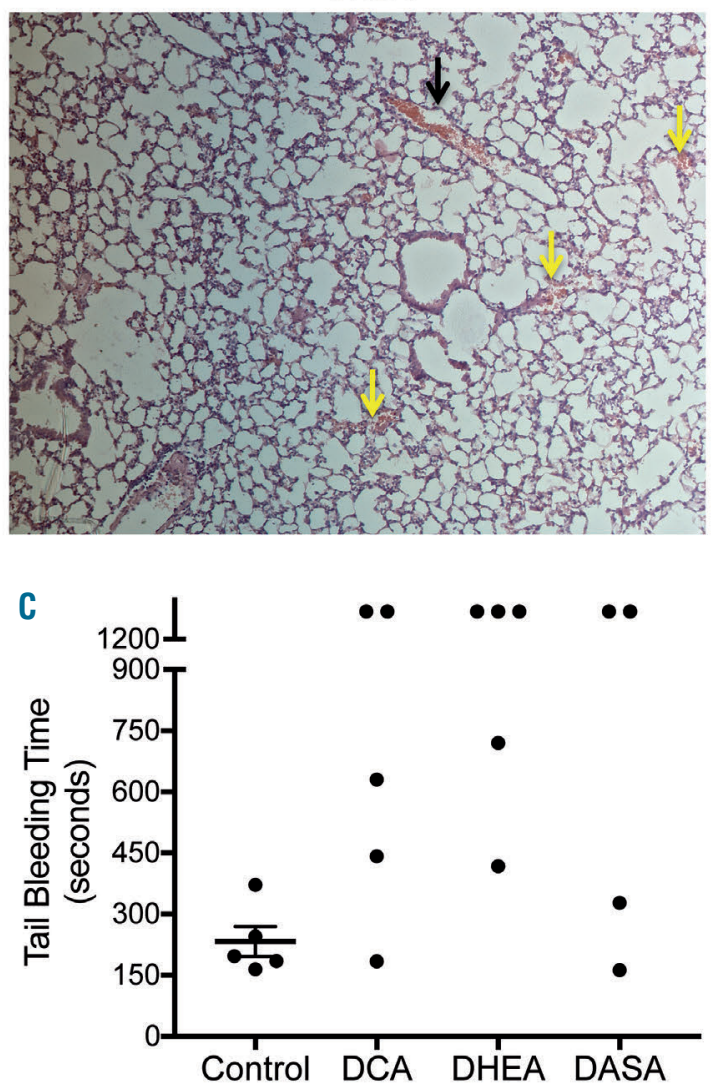

Figure 5. Small-molecule inhibitors of aerobic glycolysis and the pentose phosphate pathway impair pulmonary embolism and hemostasis. (A) Representative light microscopy images (100X magnification) of hematoxylin \& eosin-stained lung sections from mice administered collagen (1 mg/kg) plus epinephrine (10 $\mu \mathrm{g} / \mathrm{kg})$ after pre-treatment with vehicle (control), DCA, DHEA, or DASA as indicated. Black arrows indicate thrombi within the lumen of pulmonary vessels. Yellow arrows indicate extravasated erythrocytes. (B and C) Scatter dot plots showing number of thrombosed pulmonary vessels per low power field and tail-bleeding times, respectively, of mice administered with vehicle, DCA, DHEA or DASA. Data are presented as the mean \pm standard error of mean. Each dot represents an independent observation. DASA: diarylsulfonamide; DCA: dichloroacetate; DHEA: dehydroepiandrosterone sulphate. 
ities, both early and late, which are categorically energyintensive ${ }^{5,6}$ These activities lead to the generation of macroscopic cell-cell aggregates and fibrin-rich thrombi that can stop bleeding or potentially occlude blood vessels with disastrous consequences to human health. Evidently, platelet energy metabolism sustains a thrombus until it is lysed by the fibrinolytic system. Hence, in our search for an effective anti-thrombotic strategy, we focused on metabolic adaptations of platelets to agonist stimulation. We reasoned that disruption of key metabolic steps would prevent platelet activation, which could be developed into a potent anti-thrombotic measure.

When we exposed platelets to physiological agonists, there was sharp rise in cellular oxygen consumption apparently driven by energy-demanding early platelet responses such as shape change, cytoskeletal reorganization, aggregation and granule secretion. ${ }^{4,73,34}$ Intriguingly, this rise was short-lived and was followed by an abrupt drop at a time when platelets continued to aggregate and would possibly be required to discharge resource-intensive late responses such as retraction of fibrin clot, shedding of extracellular vesicles and protein synthesis. We sought the reason behind this rapid plunge in oxygen consumption despite enhanced ATP requirement. Aggregate formation could restrict access of oxygen to cells persisting within the core of the aggregate mass. However, this possibility was ruled out as oxygen flux in cells pre-treated with RGDS, a tetrapeptide that blocks platelet aggregation, was identical to that in aggregated platelets. Possible mitochondrial dysfunction in stimulated cells was also unlikely as leak respiration and non-mitochondrial oxygen consumption in oligomycin- and antimycin A-treated activated platelets, respectively, were suggestive of viable and well-coupled mitochondria. This prompted us to hypothesize a Warburg effect-like phenomenon in stimulated platelets whereby pyruvate is prevented from oxidation in the TCA cycle leading to a decline in mitochondrial respiration. Consistent with this possibility, the drop in oxygen flux was obviated when platelets were exposed to agents such as DCA (which enhances flux through the TCA cycle), or alternative electron carriers (methylene blue and toluidine blue $\mathrm{O})$.

The Warburg effect or aerobic glycolysis would require enhanced uptake of glucose by the cells in order to sustain

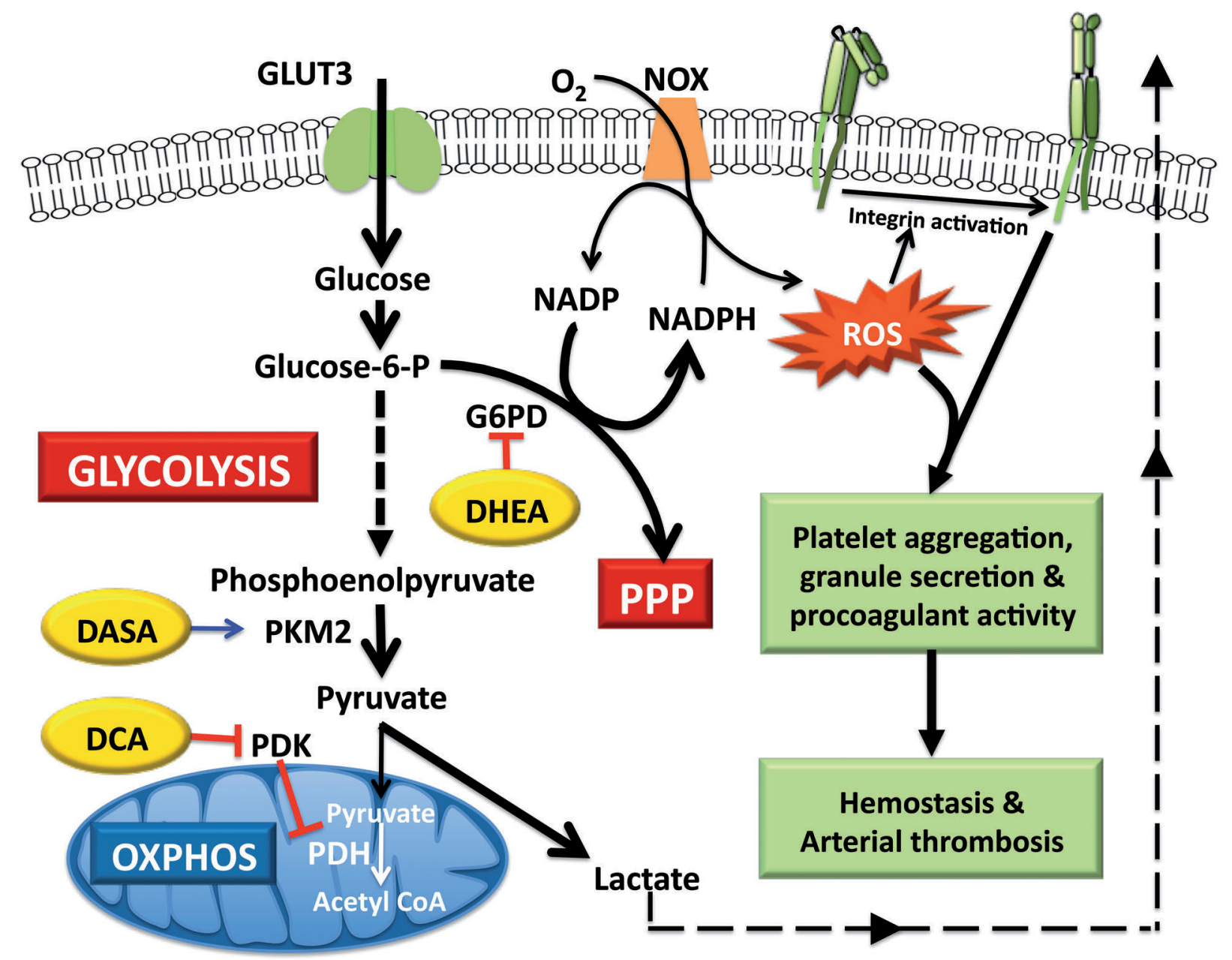

Figure 6. Scheme for metabolic flux in stimulated platelets and sites of action of small-molecule modulators. Stimulated platelets switch to aerobic glycolysis through negative regulation of pyruvate kinase $\mathrm{M} 2$ and pyruvate dehydrogenase enzyme activities. The consequent increase in flux through the pentose phosphate pathway generates NADPH that fuels ROS generation by NADPH oxidase. ROS signaling in turn mediates platelet activation, thrombosis and hemostasis. Small-molecule modulators that reverse this metabolic adaptation inhibit platelet activation and impair thrombus formation. DASA: diarylsulfonamide; DCA: dichloroacetate; DHEA: dehydroepiandrosterone sulphate; NOX: NADPH oxidase; PDH: pyruvate dehydrogenase; PDK: pyruvate dehydrogenase kinase PKM2: pyruvate kinase M2; PPP: pentose phosphate pathway; ROS: reactive oxygen species. 
adequate ATP generation by glycolysis. In keeping with this and an earlier report, ${ }^{20}$ we observed surface translocation of cytosolic GLUT3, the major glucose transporter, in thrombin-stimulated platelets. As already observed for neuronal cells, ${ }^{21}$ GLUT3 externalization in platelets was facilitated by AMPK, whose activity is known to be upregulated by agonist stimulation. ${ }^{24}$ Enhanced surface expression of GLUT3 was corroborated by a nearly 2 -fold rise in the uptake of glucose in thrombin-activated platelets. In parallel there was enhanced secretion of lactate into medium, which was in line with earlier reports of extracellular acidification induced by agonists. ${ }^{34-37}$

The metabolic checkpoint at the level of pyruvate, which is generated by the enzymatic activity of pyruvate kinase, determines whether glucose will be catabolized to lactate (by glycolysis) or through mitochondrial pathways. The enzyme PDH, part of a larger complex, catalyzes conversion of pyruvate to acetyl-CoA, which is then channeled into the TCA cycle for further oxidation and generation of ATP. Reactions catalyzed by $\mathrm{PDH}$ and pyruvate kinase are thus two critical hubs in the landscape of cellular glucose metabolism. PDH activity is regulated through inhibitory serine-293 phosphorylation by the enzyme $\mathrm{PDK}^{22}$ An increase in the activity of PDK and/or reduced $\mathrm{PDH}$ activity restricts flux of pyruvate into the TCA cycle and favors aerobic glycolysis. Inhibitory phosphorylation of $\mathrm{PDH}$ is known to be induced by AMPK under conditions of nutrient-deprivation..$^{23} \mathrm{We}$ observed an increase in phosphorylated PDH in agonist-stimulated platelets, which was reversed by inhibition of AMPK activity. Notably, we also discovered that platelets express PKM2, a tissue-specific isoform of pyruvate kinase, which, in low-activity state, facilitates aerobic glycolysis. ${ }^{38}$ Phosphorylation of PKM2 at Y105 is associated with sustenance of a dimer state with attenuated catalytic activity. ${ }^{26}$ Interestingly, agonist stimulation of platelets also led to increased expression of phosphorylated PKM2, which could be reduced to a basal level by inhibition of Src family kinases. Thus, we show here that metabolism in stimulated platelets switches to aerobic glycolysis through active regulation of $\mathrm{PDH}$ and $\mathrm{PKM} 2$; this switch leads to accumulation of glycolytic intermediates upstream of pyruvate and facilitates flux through the PPP. ${ }^{25}$ Consistent with this we observed significant enrichment in the pool of NADPH, a direct readout of the PPP, in thrombin-stimulated platelets.

Increased levels of NADPH in most cells lead to a reductive state through generation of reduced glutathione..$^{25}$ Yet, in an apparent paradox, NADPH can also serve as a substrate for the enzyme NADPH oxidase generating superoxide free radicals in the phagocytic cells, such as neutrophils, of the innate immune system..$^{39}$ Platelets are now widely recognized to be key players in immune responses. ${ }^{40,41}$ Possibly owing to genealogical and now increasingly recognized functional relationships of platelets with innate immunity, these cells, too, express significant levels of NOX activity, ${ }^{28}$ which is in fact the primary source of ROS in activated platelets..$^{27,28}$ Our findings were consistent with a rise in the levels of NADPH in stimulated platelets serving to increase the generation of ROS through activity of NOX. There is now compelling evidence indicating that NOX-generated ROS are important mediators of platelet activation signaling, ${ }^{29,42}$ which culminates in the expression of a conformationally active form of integrins $\alpha_{\mathrm{IIb}} \beta_{\mathrm{IIIa}}{ }^{27,43}$ Remarkably, pre-treatment of platelets with small-molecule inhibitors of either aerobic glycolysis (DCA or DASA) or the PPP (DHEA) brought about a significant drop in agonist-induced ROS production as well as integrin activation, and was associated with profound impairment in platelet responses to agonists (platelet aggregation, fibrinogen binding and secretion of contents of dense and $\alpha$ granules). The metabolic inhibitors also attenuated phosphatidylserine exposure and extracellular vesicle release, both measures of a procoagulant phenotype $\mathrm{s}^{31,32}$ in platelets, thus underlining the significance of aerobic glycolysis and the PPP-NOX axis in platelet activation and thrombosis. In agreement with our findings, genetic deficiency of glucose 6-phosphate dehydrogenase, the key regulatory enzyme in the PPP, is known to be associated with a remarkably lower risk of cardiovascular mortality. ${ }^{44,45}$ Strikingly, intravenous administration of metabolic modulators significantly delayed thrombus formation in mesenteric arterioles, retarded thrombus growth and prolonged time needed for complete vascular occlusion in murine models. Thus, our findings suggest that aerobic glycolysis and associated flux through the PPP in stimulated platelets play critical roles in arterial thrombosis and underscore the therapeutic potential of targeting metabolic pathways as a novel antithrombotic approach (Figure 6). These results are also consistent with two recent articles by Abel's group, which establish the role of glucose metabolism in platelet activation using transgenic mice with platelet-specific ablation of glucose transporters. ${ }^{36,37}$

Currently available anti-platelet agents are plagued by limited efficacy ${ }^{2}$ which could be attributed to remarkable redundancy in agonist stimuli and downstream signaling pathways that lead to platelet activation. Thus, conventional anti-platelet therapeutic regimens that target specific agonists/activation pathways may not confer absolute protection against thrombotic episodes, as platelets can still be stimulated by other potential triggers and parallel signaling inputs. It is, therefore, vital to discover novel anti-platelet strategies to address these challenges and cater to this largely unmet medical need. The small-molecule metabolic modulators employed in our study block essential metabolic checkpoints in stimulated platelets and effectively prevent platelet activation irrespective of the nature of the agonists and ensuing signaling pathways.

We validated our findings in a murine model of thrombosis in which deep vein thrombosis and pulmonary embolism were induced by intravenous administration of collagen and epinephrine. Notably, mice pre-treated with small-molecule metabolic modulators were protected from pulmonary thromboembolism. However, lung sections in these animals revealed extravasated erythrocytes, which was in keeping with prolonged tail-bleeding following administration of metabolic modulators. Thus, these small molecules can impair hemostasis despite conferring significant protection against thrombosis. Therefore, targeting platelet metabolism, much like currently available anti-platelet regimens, would involve a tightrope-walk of dose adjustment for effective prevention of thrombotic events without raising the risk of bleeding complications. Remarkably, metabolic modulators employed in our study have some differential effects on tail bleeding and thrombus stability, which raises hope for preferential targeting of thrombosis over hemostasis in the future by tweaking specific metabolic pathways. 
While it would not be possible to predict the off-target adverse effects of the small-molecule metabolic modulators at this stage, the potential for non-selective effects being beneficial to overall cardiovascular health cannot be overemphasized. DCA has been found to be effective in improving cardiac function after ischemia/reperfusion injury ${ }^{46-48}$ as well as in preventing restenosis after vessel injury $^{49}$ in preclinical models. There is substantial clinical evidence linking higher serum DHEA levels to decreased cardiovascular mortality. ${ }^{50}$ Small-molecule activators of PKM2 have been shown to reverse the pro-inflammatory phenotype of monocytes/macrophages isolated from patients with atherosclerotic coronary artery disease. ${ }^{51}$

Although DASA is only in preclinical stages of development as an antineoplastic drug, ${ }^{22}$ DCA is already under clinical trials against various cancers ${ }^{10,53}$ and congenital lactic acidosis ${ }^{8,54}$ while DHEA was recently granted Food and Drug Administration approval for clinical use in postmenopausal women. ${ }^{9}$ Hence, considerable information on the safety and pharmacokinetics of the latter drugs in humans is already available, which could in future pave the way for clinical trials of the drugs as potential anti- platelet/anti-thrombotic agents in the management of thrombotic disorders. In conclusion, this study suggests an indispensable role for platelet energy metabolism in thrombogenesis with potential implications for the development of anti-thrombotic strategies. Future investigations directed at metabolic adaptations of platelets within a developing thrombus in vivo could potentially identify many more therapeutic opportunities.

\section{Acknowledgments}

This research was supported by a JC Bose fellowship and grants received by $D$. Dash from the Department of Biotechnology (DBT), Science and Engineering Research Board (SERB) and Department of Science and Technology (DST), Government of India, the Indian Council of Medical Research (ICMR) and the Council of Scientific and Industrial Research (CSIR). A.T. and D.G. are recipients of UGC-JRF and CSIR$J R F$, respectively. We are grateful to the technical staff for their assistance in performing the histopathological analysis of murine lung tissue. We thank Pradyumna Kulkarni for proofreading the manuscript. D.D. acknowledges assistance from the Humboldt Foundation, Germany.

\section{References}

1. Murray CJ, Lopez AD. Measuring the global burden of disease. N Engl J Med. 2013;369 (5):448-457.

2. Franchi F, Angiolillo DJ. Novel antiplatelet agents in acute coronary syndrome. Nat Rev Cardiol. 2015;12(1):30-47.

3. Furie B, Furie BC. Mechanisms of thrombus formation. N Engl J Med. 2008;359(9):938949

4. Holmsen H, Kaplan KL, Dangelmaier CA. Differential energy requirements for platelet responses. A simultaneous study of aggregation, three secretory processes, arachidonate liberation, phosphatidylinositol breakdown and phosphatidate production. Biochem J. 1982;208(1):9-18.

5. Holmsen H. Energy metabolism and platelet responses. Vox Sang. 1981;40 (Suppl 1):1-7.

6. Verhoeven AI, Mommersteeg ME, Akkerman JW. Metabolic energy is required in human platelets at any stage during optical aggregation and secretion. Biochim Biophys Acta. 1984;800(3):242-250

7. Verhoeven AJ, Mommersteeg ME, Akkerman JW. Quantification of energy consumption in platelets during thrombininduced aggregation and secretion. Tight coupling between platelet responses and the increment in energy consumption. Biochem J. 1984;221(3):777-787

8. Stacpoole PW, Kerr DS, Barnes C, et al. Controlled clinical trial of dichloroacetate for treatment of congenital lactic acidosis in children. Pediatrics. 2006;117(5):1519-1531.

9. Labrie F, Archer DF, Koltun W, et al. Efficacy of intravaginal dehydroepiandrosterone (DHEA) on moderate to severe dyspareunia and vaginal dryness, symptoms of vulvovaginal atrophy, and of the genitourinary syndrome of menopause. Menopause. 2016;23(3):243-256.

10. Michelakis ED, Sutendra G, Dromparis P, et al. Metabolic modulation of glioblastoma with dichloroacetate. Sci Transl Med. 2010;2(31):31ra34.

11. Berendzen K, Theriaque DW, Shuster J, Stacpoole PW. Therapeutic potential of dichloroacetate for pyruvate dehydrogenase complex deficiency. Mitochondrion. 2006;6 (3):126-135.

12. Sonkar VK, Kulkarni PP, Dash D. Amyloid beta peptide stimulates platelet activation through RhoA-dependent modulation of actomyosin organization. FASEB J. 2014;28(4):1819-1829.

13. Gross MI, Demo SD, Dennison JB, et al Antitumor activity of the glutaminase inhibitor CB-839 in triple-negative breast cancer. Mol Cancer Ther. 2014;13(4):890 901.

14. Singh SK, Singh MK, Kulkarni PP, Sonkar VK, Gracio JJ, Dash D. Amine-modified graphene: thrombo-protective safer alternative to graphene oxide for biomedical applications. ACS Nano. 2012;6(3):2731-2740.

15. Nayak MK, Kulkarni PP, Dash D. Regulatory role of proteasome in determination of platelet life span. J Biol Chem. 2013;288 (10):6826-6834

16. Prakash P, Kulkarni PP, Chauhan AK. Thrombospondin 1 requires von Willebrand factor to modulate arterial thrombosis in mice. Blood. 2015;125(2):399-406.

17. Prakash P, Kulkarni PP, Lentz SR, Chauhan AK. Cellular fibronectin containing extra domain A promotes arterial thrombosis in mice through platelet Toll-like receptor 4 . Blood. 2015;125(20):3164-3172

18. Jobe SM, Wilson KM, Leo L, et al. Critical role for the mitochondrial permeability transition pore and cyclophilin D in platelet activation and thrombosis. Blood. 2008;111(3): 1257-1265.

19. Warburg $O$. On the origin of cancer cells. Science. 1956;123(3191):309-314

20. Sorbara LR, Davies-Hill TM, Koehler-Stec EM, Vannucci SJ, Horne MK, Simpson IA.
Thrombin-induced translocation of GLUT3 glucose transporters in human platelets. Biochem J. 1997;328(Pt 2):511-516.

21. Weisova P, Concannon CG, Devocelle M Prehn JH, Ward MW. Regulation of glucose transporter 3 surface expression by the AMP-activated protein kinase mediates tolerance to glutamate excitation in neurons. J Neurosci. 2009;29(9):2997-3008.

22. Holness MJ, Sugden MC. Regulation of pyruvate dehydrogenase complex activity by reversible phosphorylation. Biochem Soc Trans. 2003;31(Pt 6):1143-1151.

23. Wu CA, Chao Y, Shiah SG I in WW. Nutrient deprivation induces the Warburg effect through ROS/AMPK-dependent activation of pyruvate dehydrogenase kinase. Biochim Biophys Acta. 2013;1833(5):11471156.

24. Randriamboavoniy V, Isaak J, Fromel T, et al AMPK alpha2 subunit is involved in platelet signaling, clot retraction, and thrombus stability. Blood. 2010;116(12):2134-2140.

25. Cairns RA, Harris IS, Mak TW. Regulation of cancer cell metabolism. Nat Rev Cancer 2011;11(2):85-95.

26. Hitosugi T, Kang S, Vander Heiden MG, et al. Tyrosine phosphorylation inhibits PKM2 to promote the Warburg effect and tumor growth. Sci Signal. 2009;2(97):ra73.

27. Begonja AJ, Gambaryan S, Geiger J, et al Platelet $\mathrm{NAD}(\mathrm{P}) \mathrm{H}$-oxidase-generated ROS production regulates alphallbbeta3-integrin activation independent of the NO/cGMP pathway. Blood. 2005;106(8):2757-2760

28. Seno $\mathrm{T}$, Inoue $\mathrm{N}$, Gao D, et al. Involvement of NADH/NADPH oxidase in human platelet ROS production. Thromb Res. 2001;103(5):399-409.

29. Krotz F, Sohn HY, Pohl U. Reactive oxygen species: players in the platelet game. Arterioscler Thromb Vasc Biol. 2004;24 (11):1988-1996.

30. Bertoni A, Rastoldo A, Sarasso C, et al Dehydroepiandrosterone-sulfate inhibits 
thrombin-induced platelet aggregation. Steroids. 2012;77(3):260-268.

31. Lentz BR. Exposure of platelet membrane phosphatidylserine regulates blood coagulation. Prog Lipid Res. 2003;42(5):423-438.

32. Owens AP, 3rd, Mackman N. Microparticles in hemostasis and thrombosis. Circ Res. 2011;108(10):1284-1297.

33. Verhoeven AJ, Mommersteeg ME, Akkerman JW. Comparative studies on the energetics of platelet responses induced by different agonists. Biochem J. 1986;236(3): 879-887.

34. Akkerman JW, Holmsen H. Interrelationships among platelet responses: studies on the burst in proton liberation, lactate production, and oxygen uptake during platelet aggregation and $\mathrm{Ca} 2+$ secretion. Blood. 1981:57(5):956-966.

35. Ravi S, Chacko B, Sawada H, et al. Metabolic plasticity in resting and thrombin activated platelets. PloS one. 2015;10(4): e0123597.

36. Fidler TP, Campbell RA, Funari $T$, et al. Deletion of GLUT1 and GLUT3 reveals multiple roles for glucose metabolism in platelet and megakaryocyte function. Cell Rep. 2017;20(9):2277.

37. Fidler TP, Middleton EA, Rowley JW, et al. Glucose transporter 3 potentiates degranulation and is required for platelet activation. Arterioscler Thromb Vasc Biol. 2017;37(9): 1628-1639.

38. Mazurek S. Pyruvate kinase type M2: a key regulator of the metabolic budget system in tumor cells. Int J Biochem Cell Biol. 2011;43(7):969-980.
39. Lambeth JD, Neish AS. Nox enzymes and new thinking on reactive oxygen: a doubleedged sword revisited. Annu Rev Pathol. 2014;9:119-145.

40. Semple JW, Italiano JE Jr., Freedman J. Platelets and the immune continuum. Nat Rev Immunol. 2011:11(4):264-274.

41. Herter JM, Rossaint J, Zarbock A. Platelets in inflammation and immunity. J Thromb Haemost. 2014:12(11):1764-1775.

42. Dayal S, Wilson KM, Motto DG, Miller FJ, Ir., Chauhan AK, Lentz SR. Hydrogen peroxide promotes aging-related platelet hyperactivation and thrombosis. Circulation. 2013;127(12):1308-1316.

43. Jang JY, Min JH, Chae YH, et al. Reactive oxygen species play a critical role in collagen-induced platelet activation via SHP-2 oxidation. Antioxid Redox Signal. 2014;20 (16):2528-2540

44. Cocco P, Fadda D, Schwartz AG. Subjects expressing the glucose-6-phosphate dehydrogenase deficient phenotype experience a lower cardiovascular mortality. OJM 2008;101(2):161-163

45. Cocco P, Todde P, Fornera S, Manca MB, Manca P, Sias AR. Mortality in a cohort of men expressing the glucose-6-phosphate dehydrogenase deficiency. Blood. 1998;91 (2):706-709.

46. Barak C, Reed MK, Maniscalco SP, Sherry $\mathrm{AD}$, Malloy CR, Jessen ME. Effects of dichloroacetate on mechanical recovery and oxidation of physiologic substrates after ischemia and reperfusion in the isolated heart. J Cardiovasc Pharmacol. 1998;31(3): 336-344.
47. Bersin RM, Stacpoole PW. Dichloroacetate as metabolic therapy for myocardial ischemia and failure. Am Heart J. 1997;134(5 Pt 1):841-855

48. Wargovich TJ, MacDonald RG, Hill JA, Feldman RL, Stacpoole PW, Pepine CJ. Myocardial metabolic and hemodynamic effects of dichloroacetate in coronary artery disease. Am J Cardiol. 1988;61(1):65-70.

49. Deuse $T$, Hua $X$, Wang $D$, et al. Dichloroacetate prevents restenosis in preclinical animal models of vessel injury. Nature. 2014;509(7502):641-644

50. Wu TT, Chen Y, Zhou Y, et al. Prognostic value of dehydroepiandrosterone sulfate for patients with cardiovascular disease: a systematic review and meta-analysis. J Am Heart Assoc. 2017;6(5):e004896.

51. Shirai T, Nazarewicz RR, Wallis BB, et al. The glycolytic enzyme PKM2 bridges metabolic and inflammatory dysfunction in coronary artery disease. J Exp Med. 2016;213(3): 337-354.

52. Anastasiou D, Yu Y, Israelsen WJ, et al Pyruvate kinase M2 activators promote tetramer formation and suppress tumorigenesis. Nat Chem Biol. 2012;8(10):839-847.

53. Dunbar EM, Coats BS, Shroads AL, et al Phase 1 trial of dichloroacetate (DCA) in adults with recurrent malignant brain tumors. Invest New Drugs. 2014;32(3):452464.

54. Stacpoole PW, Gilbert LR, Neiberger RE, et al. Evaluation of long-term treatment of children with congenital lactic acidosis with dichloroacetate. Pediatrics. 2008;121(5) e1223-1228. 